\title{
Síndrome metabólico en pacientes con lupus eritematoso sistémico en el servicio de medicina interna del Hospital Eugenio Espejo
}

Torres Morales Desiré [2[, Simancas-Racines Daniel [1], Pérez-Tasigchana Raul [1] , Gavilanez Morales Claudia [1], Masson-Palacios María [1]

1 Centro de Investigación de Salud Pública y Epidemiología Clínica (CISPEC). Universidad UTE. Quito-Ecuador.

2 Médico Internista, Hospital Enrique Garcés. Quito-Ecuador.

Doi: https://doi.org/10.23936/pfr.v6i3.217

PRÁCTICA FAMILIAR RURAL | Vol.6 | No.3 | Noviembre 2021 | Recibido: 19/10/2021 | Aprobado: 24/11/2021

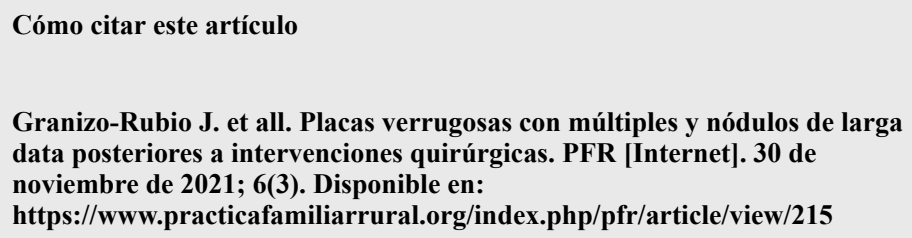

Compartir en:

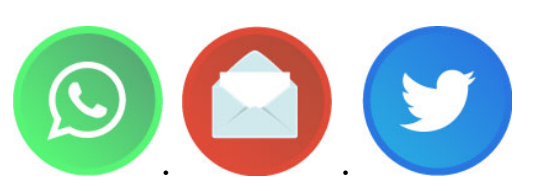

\section{Resumen}

Objetivo: Los pacientes con lupus eritematoso sistémico (LES) tienen mayor riesgo cardiovascular (RCV), esto combinado con los factores de riesgo del síndrome metabólico (SM) aumenta la probabilidad de eventos y patologías cardiacas. El presente estudio tiene como objetivo determinar la frecuencia de los factores del SM en una población de pacientes con LES.

Materiales y métodos: Se realizó un estudio descriptivo, transversal, observacional, en 132 pacientes con diagnóstico de LES del servicio de 
Medicina Interna del Hospital Eugenio Espejo, entre los meses de enero a mayo del año 2018, mediante la revisión de historias clínicas y entrevistas personales.

Resultados: Se estableció una prevalencia de SM del $30 \%$, del cual $83.5 \%$ fueron menores de 40 años, el $84.7 \%$ presentaron sobrepeso u obesidad.

Conclusiones: Los pacientes con LES presentan un estado proinflamatorio y aterogénico, que se traduce en un riesgo aumentado de desarrollar enfermedades cardiovasculares y por tanto mayor incidencia de los factores de riesgo asociados al síndrome metabólico y por tanto una inminente morbimortalidad temprana. Se considera vital tomar acciones preventivas y terapéuticas en este grupo poblacional.

Palabras clave: lupus, síndrome metabólico, riesgo cardiovascular, enfermedad cardiovascular

\title{
Metabolic syndrome in patients with systemic lupus erythematosus in the internal medicine service of Hospital Eugenio Espejo
}

\begin{abstract}
Background: Patients with systemic lupus erythematosus (SLE) have an increased cardiovascular risk, which combined with the risk factors for metabolic syndrome (MS), increase the probability of cardiac events and pathologies. Objective: To determine the frequency of MS factors in a population of SLE patients.

Materials and methods: A descriptive, cross-sectional, observational study was carried out in 132 patients with a diagnosis of SLE from the Internal Medicine service of the Eugenio Espejo Hospital, between the months of January to May 2018.

Results: A prevalence of MS of 30\% was established, of which $83.5 \%$ were under 40 years old, $84.7 \%$ were overweight or obese.

Conclusions: Patients with SLE show a proinflammatory and atherogenic state, which translates into an increased risk of developing cardiovascular diseases, thus a higher incidence of risk factors associated with MS and an imminent early morbidity and mortality. For this reason,it is essential to take preventive and therapeutic actions in this population group.
\end{abstract}

Key words: lupus, metabolic syndrome, cardiovascular risk, cardiovascular disease 
El lupus eritematoso sistémico (LES) se define como una enfermedad autoinmune, caracterizada por activación y proliferación de linfocitos $\mathrm{T}$ y $\mathrm{B}$ policlonales, producción de autoanticuerpos y la formación de complejos inmunes que ocasionan daño en tejidos y órganos(1). Se califica como un proceso inflamatorio crónico permanente con alteración de la función del endotelio vascular y por ateroesclerosis acelerada que se traduce en un mayor riesgo cardiovascular (RCV), que puede presentar complicaciones multisistémicas(2). La principal causa de mortalidad se relaciona con enfermedades cardiovasculares. La etiología aún se desconoce pero se ha encontrado relación con varios factores desencadenantes, entre ellos, genéticos, ambientales (exposición a rayos ultravioleta, infecciones virales, químicas y endocrinas), hormonales y emocionales (3).

Un mayor RCV en los pacientes con LES es reconocido e incluye un aumento del riesgo de desarrollo de Enfermedad coronaria cardiaca (ECC) y enfermedad cerebrovascular (ECV)(4), sin embargo la utilización de escalas estándar tradicionales, como por ejemplo, el puntaje de riesgo de Framingham, no es adecuado pues subestiman significativamente el RCV en la población con LES (4). Una de las alternativas es la utilización de la evaluación de riesgo de Síndrome Metabólico (SM) como un método importante de evaluar RCV, que permite identificar los pacientes que precisan una mayor intervención para prevenir la aparición de $\operatorname{ECV}(5)$.

El síndrome metabólico (SM) es un conjunto de factores de riesgo para el desarrollo de enfermedades cardiovasculares; se caracteriza por la presencia conjunta de sobrepeso/obesidad, dislipidemia, hiperglucemia e hipertensión arterial sistémica (HAS)(3). Los pacientes con LES presentan mayor frecuencia de los factores de RCV tradicionales, como la dislipidemia común, el perfil lipídico aterogénico caracterizado por niveles elevados de colesterol total, triglicéridos, lipoproteína de baja densidad (c-LDL), y lipoproteína $\mathrm{A}$, así como niveles disminuidos de lipoproteína de alta densidad (c-HDL)(4).

Los pacientes con LES también sufren con mayor frecuencia diabetes mellitus(DM) debido a la disminución significativa de la sensibilidad a la insulina y a la alta prevalencia de síndrome metabólico, de igual manera, tienen mayor frecuencia de hipertensión arterial sistémica (HAS), tabaquismo y sedentarismo(3).

En Ecuador son escasos los estudios publicados sobre el estado epidemiológico actual del SM en pacientes con LES, por esta razón, el objetivo general de este trabajo es establecer la prevalencia de SM en pacientes con LES, así como la frecuencia de los factores de RCV que forman parte del mismo y su distribución sociodemográfica, según sexo, edad y etnia. El presente estudio es de naturaleza descriptiva, cualitativa de corte transversal, en el que se utilizó entrevista directa y la revisión de los archivos digitales de los pacientes, a fin de recolectar los datos que se presentan usando la estadística descriptiva.

\section{Materiales y métodos}

Se realizó un estudio descriptivo, transversal, observacional y retrospectivo. El universo estuvo conformado por 200 pacientes con el diagnóstico de LES del servicio de Medicina Interna del Hospital Eugenio Espejo, entre los meses de enero a mayo del año 2018. El tamaño de la muestra se obtuvo mediante un cálculo probabilístico simple aleatorizado, según el universo de pacientes $(\mathrm{N}=200)$, siendo de tipo finito y con un $\mathrm{Z}$ score de $95 \%$ de nivel de confianza, usando StatCalc de EPI INFO 7, es así como se obtuvo una muestra aleatorizada de 132 pacientes que cumplieron con los criterios de inclusión, detallados a continuación.

En cuanto a los criterios de inclusión se tomó en cuenta a los pacientes en la consulta externa y hospitalización del servicio de medicina interna del Hospital Eugenio Espejo, que cumplieron con los siguientes criterios: ser mayores de 18 años, que cumplan con criterios diagnósticos de 
LES y que acepten voluntariamente ser parte del estudio. Se excluyeron a los pacientes menores de 18 años, que no cumplan criterios diagnósticos de LES, a quienes tengan diagnóstico de Nefritis Lúpica o a quienes se nieguen a ser parte de este estudio.

Se revisaron los expedientes clínicos, buscando los pacientes con diagnóstio de HAS o DM2, se seleccionó a los posibles participantes y en consulta externa se informó del estudio y se consultó su deseo por participar, una vez que los pacientes aceptaron participar se realizó una entrevista con el paciente en donde se midió peso y talla para cálculo de IMC (indice de masa corporal), además se tomó medidas de la circunferencia abdominal, posteriormente se revisó el sistema infomático HOSVITAL y de laboratorio SIEXLAB para extraer los datos necesarios como antecedentes, segumiento y exámenes de laboratorio pertinentes.

Los datos obtenidos durante el estudio fueron organizados mediante la herramienta digital Google Forms, para posteriormente convertirlos en formato de hoja de cálculo de Excel y finalmente a una base de datos mediante el programa estadístico SPSS versión 24.

\section{Resultados}

El análisis se efectuó en 132 pacientes que cumplieron con los criterios de inclusión y de exclusión del estudio y que contaban con la información completa para ingresar las variables de interés. Se utilizó la estadística descriptiva para los análisis estadísticos, usando porcentajes y proporciones para las variables cualitativas. Las asociaciones y diferencias en la distribución se calcularon mediante chi cuadrado. Los análisis se llevaron a cabo en el paquete estadístico SPSS 24.

Tabla 1. Datos demográficos

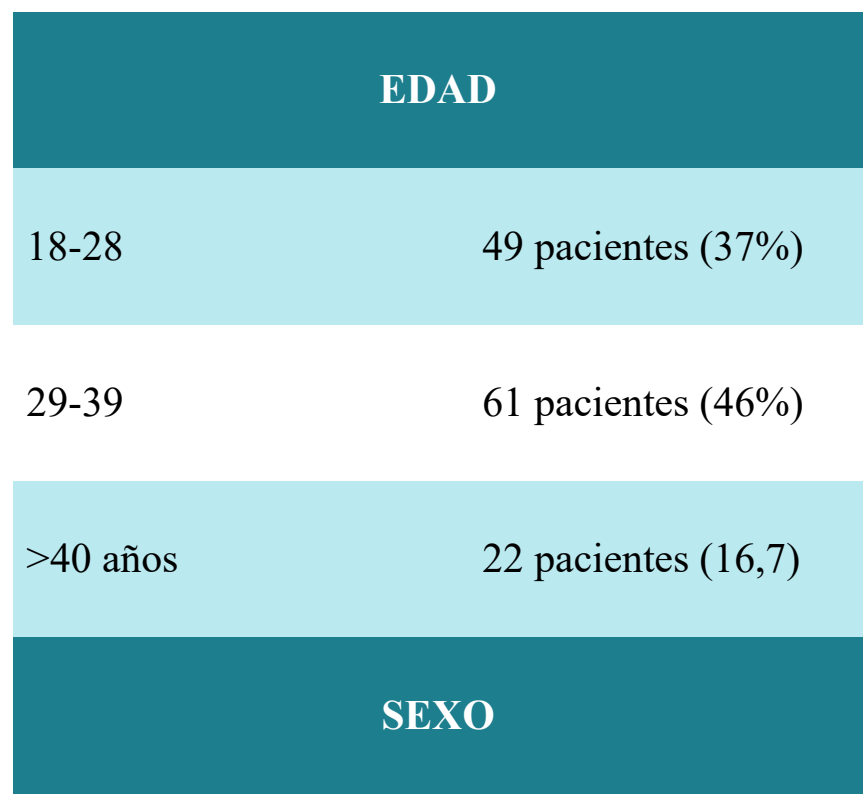

Hombres

$7(5,3 \%)$

Mujeres

$125(97,4 \%)$

\section{ETNIA}


Afroamericanos $\quad 5(3,8)$

Como resultados se obtuvo que del total de nuestra muestra $(n=132)$, el $94.7 \%$ de la población estudiada fueron de sexo femenino; el $96 \%$ de etnia mestiza, mientras que solo el $3.8 \%$ eran afroecuatorianos; el 46\% de los pacientes estaban entre la edad de 29-39 años, seguido del 37\% entre los 18 a 28 años y el $16.7 \%$ eran mayores de 40 años.

Tabla 2. Frecuencia de presentación de los componentes del síndrome metabólico

COMPONENTES DEL
SINDROME METABOLICO

La prevalencia de SM en la muestra de 132 pacientes con diagnóstico de LES del servicio de Medicina Interna del Hospital Eugenio Espejo, fue de un $30.3 \%(\mathrm{~N}=90)$. De los componentes del SM la hipertrigliceridemia fue el más frecuente en los pacientes con LES con un $49 \%$, seguido de niveles bajos de c-HDL con $40 \%$. En tercer lugar, estuvo la obesidad abdominal con una prevalencia del $38.6 \%$. En cuanto a la Hipertensión, se observó una frecuencia del $29.5 \%$. Y en último lugar se encontró la hiperglicemia con apenas un $10.6 \%$

En relación a la distribución del SM en nuestra población estudiada, logramos establecer que de los pacientes con LES que cumplieron criterios para SM, la mayoría fueron mujeres, sin embargo, cabe recordar que el $94.7 \%$ de paciente incluidos en el universo fueron del género mencionado, lo que podría significar un sesgo, lo mismo sucedió en relación a la etnia. El SM fue más frecuente en los sujetos con una edad comprendida entre los 29 y 39 años con un $52 \%$, y menos frecuente en sujetos más jóvenes, con una frecuencia observada del $22 \%$. 
La distribución del estado nutricional se asoció con la presencia de SM, así tener sobrepeso y obesidad fue más frecuente en aquellos sujetos con SM (84.7\%), En los pacientes lúpicos con SM, el $60 \%$ se encontraban con dosis intermedias de prednisona $(5-20 \mathrm{mg})$; mientras que el 30\% estaban siendo manejados con dosis de hasta $5 \mathrm{mg}$ y solo un $10 \%$ se encontraban con dosis mayores de $20 \mathrm{mg}$ al día. Por otra parte, de todos los pacientes con SM el 90\% presentaban una evolución desde el diagnóstico de LES mayor de 1 año.

Finalmente, al analizar la distribución de los componentes del SM individualmente, encontramos que la presencia de triglicéridos altos es la más frecuente en un $95 \%$, seguido de la obesidad abdominal con $85 \%$ y el c-HDL bajo $82,5 \%$, en cuarto lugar, estaba la tensión arterial elevada con el $57,5 \%$ y finalmente la hiperglicemia con el $32,5 \%$.

\section{Discusión}

El síndrome metabólico es uno de los problemas de salud pública más importantes del nuevo siglo, considerado por algunos autores como una epidemia mundial. Su prevalencia a nivel mundial se estima entre $<10 \%$ y $40 \%(6,7)$. En Latinoamérica, se reportan valores de $27 \%$ en México y 40,2 \% en Bolivia; en Ecuador es de $27.7 \%(6) . U n$ estudio realizado en una universidad del Ecuador, reportó que el componente del síndrome metabólico más frecuente fue obesidad en las mujeres $(98.5 \%)$ y los hombres $(80.0 \%)$. Junto con la obesidad y la diabetes, las combinaciones más comunes de los componentes del síndrome fueron con HDL colesterol disminuido (65\% vs. 33.3\%), hipertensión arterial $(55.1 \%$ vs. $40.0 \%)$ e hipertrigliceridemia (56.5\% vs.26.2\%), en las mujeres y los hombres respectivamente(8) (b).

En el presente estudió se observó una prevalencia del 30\% de SM en la población de los pacientes lúpicos, lo cual está dentro del intervalo mencionado en otros estudios, como los realizados en China econtrando una prevalencia de 16\% (9)y el 38\% en Puerto Rico(10).

Aunque otros estudios reportaron una mayor frecuencia, el 34\% en una población de 117 pacientes con LES en China(11), que es significativamente mayor en comparación a lo encontrado por Mok et al., y está más acorde a las cifras encontradas en este estudio y el realizado en Puerto Rico.

En el continente latinoamericano, en Argentina, con una muestra de pacientes $(n=147)$ usando los lineamientos de la Asociación Americana del Corazón (AHA/NHLBI) se obtuvo una prevalencia del $(28.6 \%)(12)$.Mientras que en Brasil se tienen datos de una prevalencia importante del 45,2\% usando la definición del Panel de Tratamiento de Adultos III (ATP- III) (13), la cual es la prevalencia más alta encontrada en los último años, la frecuencia de cada uno de los componentes del SM en los pacientes con LES también fue analizada, encontrando que los niveles bajos de c-HDL se observaron en el $84 \%$ del paciente, seguido de la HTA y la obesidad abdominal con un $80 \%$, la hipertrigliceridemia significó un $74.2 \%$ del total de los casos y finalmente la DM2 un $42 \% .(13)$

En relación a la frecuencia de los diferentes componentes del SM en los pacientes con LES, nosotros encontramos una mayor prevalencia de alteraciones lipídicas, tanto hipertrigliceridemia como niveles bajos de c-HDL, seguidos de obesidad abdominal, hipertensión y finalmente DM2. Acerca de estos datos es importante recordar, que uno de los criterios de exclusión de nuestro estudio fue el diagnóstico de nefritis lúpica, lo cual es importante ya que descartamos de alguna manera la sobreestimación de alteraciones lipídicas que pueden estar asociadas a enfermedad renal, por lo que más bien estas estarían asociadas a la prevalencia similar de obesidad en nuestros pacientes lúpicos. Y de la misma manera significaría que la proporción de pacientes con hipertensión encontrados en este estudio, no se deben a causas renales. 
Además, en necesario mencionar que por cumplimiento estricto de los criterios del III Panel estadounidense para el Tratamiento de Adultos del Programa Nacional de Educación sobre el Colesterol (NCEP ATP III) usados en este estudio, se adoptó el valor de hiperglucemia de más de $110 \mathrm{mg} / \mathrm{dl}$ establecido por dicha entidad, a pesar de que la definición actual de normo glucemia según la Organización Mundial de la Salud (OMS) y la Asociación Americana de la Diabetes (ADA) es menor a $100 \mathrm{mg} / \mathrm{dl}$. Cabe también mencionar, que en el universo estudiado no se presentaron pacientes que tuvieran valores glucémicos al límite de los cortes mencionados, de hecho, presentaron glucemias mayores de 200, lo que facilito su definición como una verdadera alteración hiperglucémica o DM2.

En relación a la hipertensión como factor frecuente en los pacientes lúpicos con SM, el estudio realizado en México por Aguilar-Salinas et al, encontró una frecuencia del $61.8 \%$ de pacientes que se encontraban recibiendo tratamiento antihipertensivo así como con mediciones de presión arterial mayores de 140/90(14). Sin embargo, cabe recalcar que en este estudio el universo incluyó paciente con nefritis lúpica, lo cual podría sobreestimar su prevalencia.

En cuanto a la dislipidemia, un estudio realizado en Hungría se menciona una prevalencia tanto de niveles bajos de c-HDL, como hipertrigliceridemia del 30\%, la cual podía llegar hasta el $60 \%$ a los 3 años de diagnóstico de LES(15), valores que coinciden con lo observado en este estudio.

Mientras que otros autores han encontrado asociación entre dosis altas de prednisona así como con evolución de más de un año de la enfermedad, los datos obtenidos en nuestro estudio no fueron estadísticamente significativos para estas variables, sin embargo es importante recordar que dichos estudios realizaron el análisis con dosis acumuladas de corticoide y la mayoría de pacientes tenían evoluciones de la enfermedad más de 7 a 10 años $(11,13)$

\section{Conclusiones}

La prevalencia de dislipidemia aterogénica fue de un 40.1 y $49.2 \%$ (niveles bajos de HDL e hipertrigliceridemia respectivamente); la de obesidad abdominal fue de $38.6 \%$; hipertensión $29.5 \%$ e hiperglicemia mayor de $200 \mathrm{mg} / \mathrm{dl}$ o DM2 fue del $10.6 \%$ en los pacientes con diagnóstico de LES del servicio de Medicina Interna del Hospital Eugenio Espejo.

El SM se presentó mayormente, con una frecuencia del $83.5 \%$ en pacientes lúpicos menores de 40 años, predominantemente en mujeres de etnia mestiza y el $84 \%$ presentaron sobrepeso u obesidad (66.7\% y $18 \%$ respectivamente).

Los pacientes con LES que cumplieron con los criterios para SM lo hicieron el $47 \%$ con 3 criterios, el $42 \%$ con 2 criterios y solo el $11 \%$ con los 5 criterios completos.

\section{Recomendaciones}

Se recomienda poner mayor énfasis en la población lúpica joven a la hora de identificar factores de riesgo metabólico cardiovascular, ya que, según lo encontrado en este estudio, esta población estaría en riesgo de ECV a pesar de no pasar de los 55 años, debido a las cifras de prevalencia reportadas de los componentes del SM, especialmente la alteración de los valores lipídicos.

Se requieren más estudios para identificar otros factores asociados al SM en nuestros pacientes con LES, como por ejemplo estudios de casos y controles en los que se pueda comparar y realizar análisis multivariables en relación a la duración de la enfermedad, Indice de actividad de la enfermedad de LES (SLEDAI), dosis acumulada de prednisona, anticuerpos, marcadores inflamatorios entre otros. 
Debería realizarse un estudio multicéntrico con todos los hospitales de Quito, o mejor aún de las grandes ciudades del Ecuador, que avalen los resultados de esta investigación para poder tener datos actualizados de SM y sus componentes diagnósticos (obesidad abdominal, hipertrigliceridemia, etc.), los mismos que puedan establecer una prevalencia mucho más acertada que la encontrada en este estudio y de esta manera ayudar a la toma de decisiones en la consulta médica.

\section{Referencias bobliográficas}

1. Olusi SO, George S. Prevalence of LDL atherogenic phenotype in patients with systemic lupus erythematosus. Vasc Health Risk Manag. 2011;7(1):75-80.

2. Rodríguez Hernández R, Diéguez Martínez M, López Báster J, Alberteris Rodríguez A, de Valle Fernández I, Miguel Soca P. Comportamiento clínico epidemiológico del síndrome metabólico en pacientes con lupus eritematoso sistémico. Rev Cuba Reumatol RCuR. 2016;18(3):2.

3. Romero-Moreno JR, Ramírez-Villafaña M, González-Ponce F, González-López L. Síndrome metabólico en lupus eritematoso sistémico. El Resid. 2014;9(3):116-23.

4. De Jesús Batún Garrido JA, Alba HAR, Núñez ÉH. Riesgo cardiovascular en lupus eritematoso sistémico. Rev Colomb Reumatol. 2016;23(4):242-9.

5. Alberti KGMM, Eckel RH, Grundy SM, Zimmet PZ, Cleeman JI, Donato KA, et al. Harmonizing the metabolic syndrome: A joint interim statement of the international diabetes federation task force on epidemiology and prevention; National heart, lung, and blood institute; American heart association; World heart federation; International . Circulation. 2009; 120(16):1640-5.

6. Buenaño Anchundia SS, Cueva Vizhñay EE. Prevalencia de síndrome metabólico y factores de riesgo asociados en una muestra de individuos adultos del Ecuador. 2020; Available from: http://repositorio.ug.edu.ec/handle/redug/49176

7. Pocoví M, Ferreira I. Fenotipo, fisiopatología y genotipo del síndrome metabólico desde la prehistoria hasta nuestros días. El síndrome metabólico. 2010. 1-40 p.

8. Trujillo R B, Barrera G F RPR. Prevalencia de síndrome metabólico en pacientes con diabetes mellitus tipo 2 residentes en la ciudad de Otavalo. 2017;6. Available from: https://revistadigital.uce.edu.ec/index.php/CIENCIAS_MEDICAS/article/view/1060

9. Mok CC, Poon WL, Lai JPS, Wong CK, Chiu SM, Wong CK, et al. Metabolic syndrome, endothelial injury, and subclinical atherosclerosis in patients with systemic lupus erythematosus. Scand J Rheumatol. 2010 Jan;39(1):42-9.

10. Negrón AM, Molina MJ, Mayor AM, Rodríguez VE, Vilá LM. Factors associated with metabolic syndrome in patients with systemic lupus erythematosus from Puerto Rico. Lupus. 2008 Apr;17(4):348-54.

11. Liu S-Y, Han L-S, Guo J-Y, Zheng Z-H, Li H, Zhang L, et al. Metabolic syndrome in Chinese patients with systemic lupus erythematosus: no association with plasma cortisol level. Lupus. 2013 Apr;22(5):519-26.

12. Bellomio V, Spindler A, Lucero E, Berman A, Sueldo R, Berman H, et al. Metabolic syndrome in Argentinean patients with systemic lupus erythematosus. Lupus. 2009 Oct;18(11):1019-25.

13. Medeiros MM das C, Xavier de Oliveira ÍM, Ribeiro ÁTM. Prevalence of metabolic syndrome in a cohort of systemic lupus erythematosus patients from Northeastern Brazil: association with disease activity, nephritis, smoking, and age. Rheumatol Int. 2016;36(1):11724.

14. Aguilar-Salinas CA, Rojas R, Gómez-Pérez FJ, Valles V, Ríos-Torres JM, Franco A, et al. High prevalence of metabolic syndrome in Mexico. Arch Med Res. 2004;35(1):76-81.

15. Szabó MZ, Szodoray P, Kiss E. Dyslipidemia in systemic lupus erythematosus. Immunol Res. 2017;65(2):543-50. 